\title{
An underlying principle for the study of circulating progenitor cells in diabetes and its complications
}

\author{
G. P. Fadini
}

Published online: 14 May 2008

(C) Springer-Verlag 2008

Keywords Diabetes mellitus · Endothelium · Progenitor cells $\cdot$ Renegeration $\cdot$ Stem cells

\author{
Abbreviations \\ EPC endothelial progenitor cell \\ KDR kinase insert domain receptor \\ SDF-1 stromal-derived factor-1
}

Diabetes mellitus profoundly affects vascular biology, and its associated complications are related to impairment of macro- and microvascular beds. The reasons for the aggressiveness of diabetic vasculopathy are incompletely understood, but it has long been established that the vascular endothelium plays a central role. Novel insights followed the discovery that a population of bone marrowderived cells is a source of endothelial cells [1]. As demonstrated experimentally, critical functions of the cardiovascular system, such as maintenance of endothelial integrity and generation of new blood vessels, involve the bone marrow through so-called endothelial progenitor cells (EPCs) (reviewed in [2]). It is not difficult to perceive how impairment of EPCs could promote the development of cardiovascular disease in its many forms. According to this hypothetical model, in patients with multiple risk factors that promote endothelial damage, a reduction in the number of EPCs would prevent endothelial repair, thus favouring the development of atherosclerotic lesions. This would be

G. P. Fadini $(\bowtie)$

Department of Clinical and Experimental Medicine,

University of Padua, Medical School,

Via Giustiniani, 2,

35100 Padua, Italy

e-mail: gianpaolofadini@hotmail.com

e-mail: gianpaolo.fadini@unipd.it followed by impaired restoration of blood flow in ischaemic tissues, as a result of defective angiogenesis, contributing to disease progression [2]. This is why the observation that type 1 and type 2 diabetic patients have dysfunctional and severely reduced EPCs has led to a novel pathogenic model of diabetic complications [3].

In this issue of Diabetologia, Egan et al. provide further demonstration of a reduction in EPC levels in unselected type 2 diabetic patients [4]. To understand why another confirmatory study was needed, we have to take a few steps backwards, because this hot topic has prompted broad discussion on ways of identifying and isolating the bloodderived progenitors [5]. EPCs were originally separated from cells expressing the stem cell antigen CD34 or the endothelial antigen known as kinase insert domain receptor (KDR) [1]. In subsequent early studies, EPCs were identified as $\mathrm{CD} 34^{+} \mathrm{KDR}^{+}$cells and measured by flow cytometry. However, CD34 is also an endothelial antigen and KDR is also expressed on haematopoietic progenitor cells. Thus, these antigens might not distinguish haematopoietic progenitor cells from endothelial progenitor cells and mature endothelium. To overcome this problem, some investigators proposed that the parallel use of the stem cell antigen CD133 could restrict the analyses to progenitor cells only, because it is never expressed on mature endothelium. Unfortunately, the $\mathrm{CD} 34^{+} \mathrm{CD} 133^{+} \mathrm{KDR}^{+}$ phenotype is quite difficult to measure reliably, and there are data (conflicting indeed) indicating that these cells are not endothelial, but haematopoietic progenitors [6, 7], likely reflecting that EPCs and blood cells have a common antecedent (the haemangioblast) in adulthood as well as during embryonic development. Other markers have been used to characterise EPCs, but none of several complex antigenic combinations can unambiguously define these cells [5]. Thus, disparate EPC phenotypes continue to be used, and results are not always consistent, such that direct 
comparison of studies is not always possible. Moreover, attributing a biological function to a relatively simple antigenic phenotype is an abstraction that may not always hold true, as highlighted in a recent commentary published in Diabetologia [8]. Unfortunately, there are also a wide variety of methods used to isolate and culture EPCs, which can produce different, even conflicting, results and interpretations [9, 10].

At this point, one might even wonder whether EPCs really exist as a unique entity and, being so elusive in nature, whether they actually play an important role in diabetic complications. There are different ways of fairly addressing these provocative issues [10]. First, experts could meet together and reach a consensus on EPC methods. Second, each research group could undertake an extensive evaluation of their 'putative EPCs' to unequivocally demonstrate endothelial differentiation in vitro and in vivo. Third, there could be a systematic assessment of multiple putative EPC phenotypes in relation to a given medical condition. This latter approach has been implemented by Egan and colleagues, who purportedly assessed many cellular phenotypes simultaneously, with the aim of documenting a definite pattern of progenitor cell reduction in diabetes. Consistent with previous studies, the number of $\mathrm{CD} 34^{+}$and $\mathrm{CD} 34^{+} \mathrm{KDR}^{+}$cells were found to be lower in diabetic than non-diabetic participants $[11,12]$, but the authors went further, showing reductions in other phenotypes, such as $\mathrm{CD} 133^{+}, \mathrm{CD} 117^{+}, \mathrm{CD} 133^{+} \mathrm{KDR}^{+}, \mathrm{CD} 34^{+}$ $\mathrm{CD} 33^{+} \mathrm{KDR}^{+}, \mathrm{CD} 117^{+} \mathrm{KDR}^{+}$and $\mathrm{CD} 34^{+} \mathrm{CD} 31^{+}$. This is in itself a major advancement, as it establishes diabetes mellitus as the only clinical condition for which a decline in circulating progenitors has been demonstrated so extensively and comprehensively. Recent results on the effects of hyperglycaemia on cultured EPCs $[13,14]$ leave no doubt as to the negative effect of diabetes on these cells. However, different progenitor phenotypes partially overlap and have different biological meanings. For example, while total $\mathrm{CD} 34^{+}$and $\mathrm{CD} 133^{+}$cells are mostly haematopoietic, cells bearing endothelial antigens have various degrees of endothelial commitment and differentiation, KDR marking immature, and CD31 more mature EPCs. To further complicate this picture, the tyrosine kinase receptor CD117 (c-kit) is also expressed on tissue-resident stem/ progenitor cells, and these cells may be found in the bloodstream as the result of an equilibrium between the circulation and tissue niches, or as a result of tissue damage [15]. Remarkably, experimental diabetic cardiomyopathy has been related to a reduction in $\mathrm{CD} 117^{+}$cardiac-resident progenitor cells [16], which can be detected in the bloodstream in certain conditions [17]. The study by Egan et al. moves away from strictly considering progenitors for the endothelial lineage towards a more general evaluation of multi-lineage progenitor cells that may have important functions in the cardiovascular system of diabetic patients.
It would be worthwhile to further investigate whether different progenitor cell phenotypes are differentially modulated in the presence of specific patterns of diabetic complications in a manner consistent with their lineage and function.

Mechanistically, the authors attributed the profound reduction in progenitor cells to impaired bone marrow mobilisation, because of the lower expression of CXCR4 in diabetic vs control peripheral blood mononuclear cells and $\mathrm{CD} 34^{+}$cells. While binding of the chemokine stromalderived factor-1 (SDF-1) to its receptor, CXCR4, is indeed involved in EPC mobilisation and homing [18], CXCR4 is cleaved after cell mobilisation, contributing to transient cell dysfunction [19]. Therefore, data on CXCR4 expression cannot be used to draw conclusions on mobilisation capacity under steady-state conditions. Moreover, the authors did not measure plasma SDF-1 concentrations, precluding a full assessment of the SDF-1-CXCR4 axis, and limiting the interpretation of the reduced CXCR4 expression in diabetes. Levels of the ligand vascular endothelial growth factor (VEGF), which binds to KDR, and soluble c-kit ligand (KitL), which binds to CD117, should also be determined, as they have mobilising activity and modulate receptor expression [20] (Fig. 1).

The extraordinarily consistent reduction of almost all these circulating cells in diabetes raises the question of whether this represents a non-specific failure of the bone marrow, rather than a targeted alteration of lineagecommitted progenitors. While this sort of bone marrow 'stunning' might therefore represent a disease epiphenomenon, we have reasons to believe that the pattern of progenitor cell demodulation in diabetes is causally related to disease complications. Nguyen et al. have shown that myofibroblast progenitor cells are increased in number in diabetic patients compared with controls [21], highlighting that such a dysfunction is selective for cardio- and vasculoprotective progenitors, while there may be a concomitant expansion of circulating cells with pro-remodelling activities. If a bone marrow defect is present, it seems to be reversible, because glucose-lowering therapies has been shown to restore the level of circulating progenitors to some extent [22-24]. Finally, as shown previously and confirmed by Egan et al., progenitor cell decline was linearly correlated with the severity of diabetic complications. All these observations do not necessarily prove that deregulation of progenitor cells promote diabetic complications, but are consistent with experimental studies suggesting protective effects of EPCs and detrimental effects of smooth muscle-like progenitors [25].

Regardless of the pathogenic implications, progenitor cell levels may be used as surrogate biomarkers in the clinical setting. Clinical studies on diabetic complications provided strong evidence of EPC alterations in the setting of macrovascular disease, while there are fewer data on 
microangiopathy, with the exception of retinopathy [3]. In conductance vessels, EPC impairment can be related to the development of atherosclerosis, while in resistance vessels it may lead to microvascular rarefaction. Remarkably, the study by Egan et al. shows that microvascular involvement was more consistently associated with the downregulation of different progenitor cell phenotypes than was the macroangiopathic burden. However, the literature still lacks specific studies on human diabetic neuropathy and nephropathy. The difficulty inherent to the design of such studies is that type 2 diabetic patients have concomitant risk factors, such as hypertension and dyslipidaemia, and often present with more than one complication and/or comorbidities. Multivariable analyses are usually performed to control for these factors, as done in this case, but the impact of such results may be limited because of colinearity and residual confounding. To gather less complicated data, selection of patients becomes critical: patients with good glycaemic control and without comorbid conditions or concomitant risk factors who develop diabetes-specific complications may provide valuable information on the role of progenitor cells in diabetic complications.

Longitudinal studies are the next step. Clinical utility would come from the observation that progenitor cell reduction predicts the development or progression of diabetic complications. For example, do EPC levels predict decline of glomerular filtration rate in microalbuminuric patients? Do EPCs predict future retinal neoangiogenesis? Given the supposed common pathogenesis between vascular disease and insulin resistance, do they even predict development of type 2 diabetes? If these questions are positively answered, and if experimental models continue to support a direct role for progenitor cell alterations in diabetic complications, we will finally be compelled to consider cell-based therapies in our patients.

Duality of interest The author declares that there is no duality of interest associated with this manuscript.

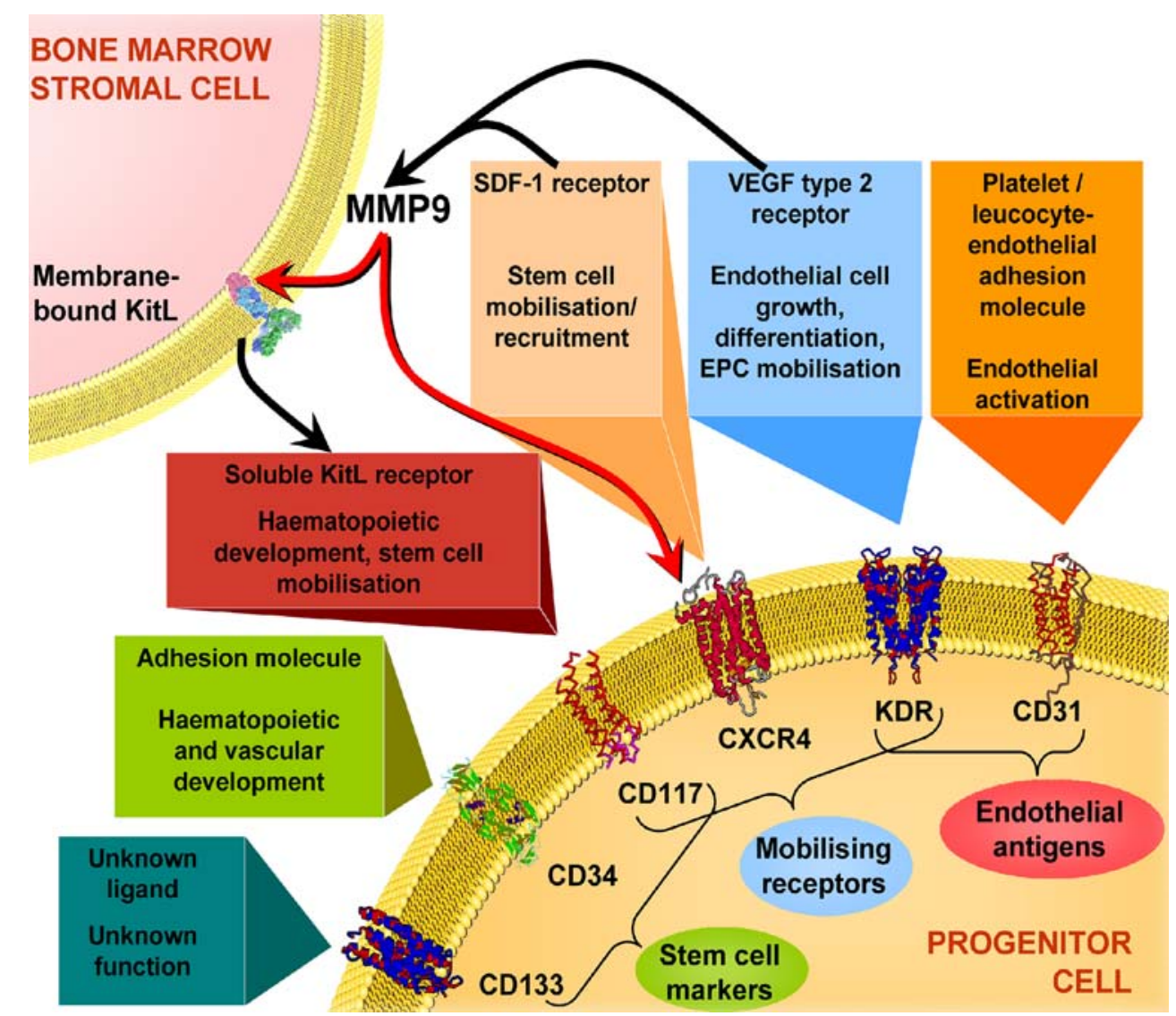

Fig. 1 The role and meaning of surface antigens in progenitor cell function and regulation. Typical antigens that characterise progenitor cells can be distinguished into stem cell markers (CD34, CD133 and CD117), mobilising receptors (CD117, CXCR4 and KDR) and endothelial markers (KDR and CD31). CD34 might also be considered an endothelial antigen. CXCR4 and KDR ligands (SDF-1 and vascular endothelial growth factor [VEGF], respectively) activate bone marrow matrix metalloproteinase (MMP)9-mediated cleavage of membrane-bound c-kit ligand (KitL). Upon binding to its receptor,

CD117, soluble KitL promotes stem/progenitor cell release into the circulation. MMP9 could digest not only membrane KitL, but also CXCR4 expressed on the surface of progenitor cells (red arrows). As a result, recruitment of progenitor cells is transiently inhibited, as long as they persist in the bloodstream. In experimental diabetes, impairment of the SDF-1-CXCR4 and VEGF-KDR axes, together with reduced bone marrow responsiveness, contribute to the decrease in circulating levels of progenitor cells [23] 


\section{References}

1. Asahara T, Murohara T, Sullivan A et al (1997) Isolation of putative progenitor endothelial cells for angiogenesis. Science 275:964-967

2. Fadini GP, Agostini C, Sartore S, Avogaro A (2007) Endothelial progenitor cells in the natural history of atherosclerosis. Atherosclerosis 194:46-54

3. Fadini GP, Sartore S, Agostini C, Avogaro A (2007) Significance of endothelial progenitor cells in subjects with diabetes. Diabetes Care 30:1305-1313

4. Egan CG, Lavery R, Caporali F et al (2008) Generalised reduction of putative endothelial progenitors and CXCR4-positive peripheral blood cells in type 2 diabetes. Diabetologia DOI 10.1007/ s00125-008-0939-6

5. Ingram DA, Caplice NM, Yoder MC (2005) Unresolved questions, changing definitions, and novel paradigms for defining endothelial progenitor cells. Blood 106:1525-1531

6. Case J, Mead LE, Bessler WK et al (2007) Human CD34 $4^{+} \mathrm{AC}_{133^{+}}$ VEGFR-2 ${ }^{+}$cells are not endothelial progenitor cells but distinct, primitive hematopoietic progenitors. Exp Hematol 35:1109-1118

7. Fadini GP, Avogaro A, Agostini C (2007) Critical assessment of putative endothelial progenitor phenotypes. Exp Hematol 35:1479-1480

8. Hanley NA (2007) Bone marrow-derived cells and the vasculature in diabetes: from biomarker to treatment. Diabetologia 50:2033-2035

9. Leor J, Marber M (2006) Endothelial progenitors. A new tower of Babel? J Am Coll Cardiol 48:1588-1590

10. Fadini GP, Baesso I, Albiero M, Sartore S, Agostini C, Avogaro A (2008) Technical notes on endothelial progenitor cells: ways to escape from the knowledge plateau. Atherosclerosis 197:496-503

11. Fadini GP, Miorin M, Facco M et al (2005) Circulating endothelial progenitor cells are reduced in peripheral vascular complications of type 2 diabetes mellitus. J Am Coll Cardiol 45:1449-1457

12. Fadini GP, Pucci L, Vanacore R et al (2007) Glucose tolerance is negatively associated with circulating progenitor cell levels. Diabetologia 50:2156-2163

13. Ingram DA, Lien IZ, Mead LE et al (2008) In vitro hyperglycemia or a diabetic intrauterine environment reduces neonatal endothelial colony-forming cell numbers and function. Diabetes 57:724-731
14. Marchetti V, Menghini R, Rizza S et al (2006) Benfotiamine counteracts glucose toxicity effects on endothelial progenitor cell differentiation via Akt/FoxO signaling. Diabetes 55:2231-2237

15. Aicher A, Rentsch M, Sasaki K et al (2007) Nonbone marrowderived circulating progenitor cells contribute to postnatal neovascularization following tissue ischemia. Circ Res 100: $581-589$

16. Rota M, LeCapitaine N, Hosoda T et al (2006) Diabetes promotes cardiac stem cell aging and heart failure, which are prevented by deletion of the p66shc gene. Circ Res 99:42-52

17. Wojakowski W, Tendera M, Michalowska A et al (2004) Mobilization of $\mathrm{CD} 34 / \mathrm{CXCR} 4^{+}, \mathrm{CD} 34 / \mathrm{CD} 117^{+}$, c-met $^{+}$stem cells, and mononuclear cells expressing early cardiac, muscle, and endothelial markers into peripheral blood in patients with acute myocardial infarction. Circulation 110:3213-3220

18. Schober A, Karshovska E, Zernecke A, Weber C (2006) SDF-1 $\alpha$ mediated tissue repair by stem cells: a promising tool in cardiovascular medicine. Trends Cardiovasc Med 16:103-108

19. Honold J, Lehmann R, Heeschen C et al (2006) Effects of granulocyte colony-stimulating factor on functional activities of endothelial progenitor cells in patients with chronic ischemic heart disease. Arterioscler Thromb Vasc Biol 26:2238-2243

20. Aicher A, Zeiher AM, Dimmeler S (2005) Mobilizing endothelial progenitor cells. Hypertension 45:321-325

21. Nguyen TQ, Chon H, van Nieuwenhoven FA, Braam B, Verhaar MC, Goldschmeding R (2006) Myofibroblast progenitor cells are increased in number in patients with type 1 diabetes and express less bone morphogenetic protein 6: a novel clue to adverse tissue remodeling. Diabetologia 49:1039-1048

22. Humpert PM, Neuwirth R, Battista MJ et al (2005) SDF-1 genotype influences insulin-dependent mobilization of adult progenitor cells in type 2 diabetes. Diabetes Care 28:934-936

23. Fadini GP, Sartore S, Schiavon M et al (2006) Diabetes impairs progenitor cell mobilisation after hindlimb ischaemia-reperfusion injury in rats. Diabetologia 49:3075-3084

24. Fadini GP, Baesso I, Agostini C et al (2007) Maternal insulin therapy increases fetal endothelial progenitor cells during diabetic pregnancy. Diabetes Care 31:808-810

25. Sata M (2003) Circulating vascular progenitor cells contribute to vascular repair, remodeling, and lesion formation. Trends Cardiovasc Med 13:249-253 